\title{
Liraglutide administration improves hormonal/metabolic profile and reproductive features in women with HAIR-AN syndrome
}

\author{
S Livadas' ${ }^{1}$, I Androulakis', N Angelopoulos', A Lytras¹, F Papagiannopoulos² and G Kassi3 \\ ${ }^{1}$ Endocrine Unit, Metropolitan Hospital, Athens, Greece, 2Novo-Nordisk, Athens, Greece, and ${ }^{3}$ Endocrine Unit, \\ Alexandra Hospital, Athens, Greece
}

Correspondence should be addressed to S Livadas

Email

sarntis@gmail.com

\begin{abstract}
Summary
HAIR-AN syndrome, the coexistence of Hirsutism, Insulin Resistance (IR) and Acanthosis Nigricans, constitutes a rare nosologic entity. It is characterized from clinical and biochemical hyperandrogenism accompanied with severe insulin resistance, chronic anovulation and metabolic abnormalities. Literally, HAIR-AN represents an extreme case of polycystic ovary syndrome (PCOS). In everyday practice, the management of HAIR-AN constitutes a therapeutic challenge with the available pharmaceutical agents. Specifically, the degree of IR cannot be significantly ameliorated with metformin administration, whereas oral contraceptives chronic administration is associated with worsening of metabolic profile. Liraglutide and exenatide, in combination with metformin, have been introduced in the management of significantly obese women with PCOS with satisfactory results. Based on this notion, we prescribed liraglutide in five women with HAIR-AN. In all participants a significant improvement regarding the degree of $I R$, fat depositions, androgen levels and the pattern of menstrual cycle was observed, with minimal weight loss. Furthermore, one woman became pregnant during liraglutide treatment giving birth to a healthy child. Accordingly, we conclude that liraglutide constitutes an effective alternative in the management of women with HAIR-AN.
\end{abstract}

\section{Learning points:}

- HAIR-AN management is challenging and classic therapeutic regimens are ineffective.

- Literally HAIR-AN syndrome, the coexistence of Hirsutism, Insulin Resistance and Acanthosis Nigricans, represents an extreme case of polycystic ovary syndrome.

- In cases of HAIR-AN, liraglutide constitutes an effective and safe choice.

\section{Background}

HAIR-AN syndrome, namely the combination of hyperandrogenism, insulin resistance and acanthosis nigricans although a rare nosologic entity, represents an extreme case of polycystic ovary syndrome (PCOS). Indeed the cardinal features of PCOS such as clinical and biochemical hyperandrogenism, severe insulin resistance, chronic anovulation and metabolic abnormalities dominate HAIR-AN phenotype. The characteristic acanthosis nigricans, namely the velvety, hyperpigmented patches of skin observed usually in the axilla or the neck of patients, should be attributed to the long-term exposure of the keratinocytes to insulin. Actually, in human keratinocytes, receptors for both insulin and insulinlike growth factors have been found on their surface. HAIR-AN management is challenging, since classic therapeutic modalities usually applied in PCOS such as metformin or oral contraceptives seem inefficacious to adequately control the symptoms. However, the advent 
of GLP-1 analogues and their successful use not only in patients with type 2 diabetes, but also in cases of PCOS with morbid obesity, guide us to the use of liraglutide in a series of women suffering from of HAIR-AN, an approach never introduced before to the best of our knowledge.

\section{Case presentation}

Five women with HAIR-AN visited our outpatient clinic due to severe chronic oligomenorrhea, accompanied with hyperandrogenic signs (acne and hirsutism) and acanthosis nigricans in both neck and the axilla. The mean age of the studied subjects were $29 \pm 2.4$ years and their mean BMI $31 \pm 3.5 \mathrm{~kg} / \mathrm{m}^{2}$.

\section{Investigation}

In all subjects a complete biochemical/hormonal profile between 3 and 7 days after a spontaneous bleeding episode and body fat analysis (total and trunk) was performed (Tanita BC 418). Additionally, an $75 \mathrm{~g}$ oral glucose tolerance test (OGTT) with estimation of insulin levels at basal and 30-, 60-, 90- and 120-min intervals was carried out. No woman reported use of any medication during the last semester that could interfere with the normal function of the hypothalamic-pituitary-gonadal axis, including metformin. The IR indices, HOMA-IR, Matsuda index and area under the curve for glucose (AUC Glucose) and Insulin (AUC Insulin) were calculated. The degree of hirsutism was evaluated with the use of Ferriman-Gallway score (FG score).

\section{Treatment}

In all patients, liraglutide at a dose $1.8 \mathrm{mg}$ /day was administered subcutaneously for a mean duration of $14 \pm 4$ months. During the study period, liraglutide was not available at the dose of $3 \mathrm{mg}$ in Greece and available studies in PCOS have used the 1.8 regimen. No other drug was prescribed to the studied subjects. None of the patients used metformin 3 months prior to treatment. Informed consent was obtained from all women and the study was approved by the institutional review board. The study met the requirements of the 1975 Helsinki guidelines.

\section{Outcome and follow-up}

In all participants, a statistical significant decrease of insulin resistance index HOMA-IR, androgen levels, with significant improvement of fat deposition, menstrual cycle pattern and the degree of hirsutism was disclosed ( $P<0.05$ for all comparisons). Furthermore, one woman became pregnant during liraglutide treatment giving birth to a healthy child. Liraglutide was discontinued when pregnancy was certified. None of the patients reported severe nausea and vomiting, and none of them discontinued liraglutide administration due to drug side effects. Pertinent data of the studied population and comparisons before and post treatment are depicted in Table 1.

\section{Discussion}

HAIR-AN, the syndrome defined by the acronyms of Hyperandrogenism, Insulin resistance and Acanthosis nigricans, is divided to type $\mathrm{A}$ and $\mathrm{B}$, with regard to whether the severe insulin resistance is either inherited (A) or acquired (B) (1). However, in both cases, acanthosis nigricans, namely velvety, hyperpigmented patches of skin is observed, especially in the neck and axilla, due to the long-term exposure of the keratinocytes to insulin (2). Hyperinsulinaemia dominates the clinical and biochemical manifestation of patients suffering from the syndrome. As it is well known from PCOS pathophysiology, insulin and androgens potentiate each other's action and thus a perpetuating vicious circle of chronic anovulation and hyperandrogenic signs followed by a male pattern fat deposition is expected in patients suffering from HAIR-AN $(3,4)$.

Liraglutide is a long-acting GLP1 analog with 97\% linear amino acid sequence homology to human GLP1 and a half-life of $13 \mathrm{~h}$. This molecule exerts pleiotropic actions in metabolism, as it increases glucose-dependent insulin secretion, decreases glucagon secretion, slows gastric emptying and increases satiety (5). Furthermore, few small studies conducted in PCOS women of either liraglutide or exenatide have shown promising results regarding the attenuation of insulin resistance, the degree of hyperandrogenemia and the restoration of menstrual cyclicity $(6,7,8,9)$. Additionally, clinical trials in diabetics have shown that liraglutide in the dose of $1.8 \mathrm{mg} /$ day effectively decreased vascular fat mass (10).

These properties suggest that liraglutide could be a strong candidate and guided our decision for its use in the management of HAIR-AN. Actually, it was revealed that long-term liraglutide administration is safe and effective in women with HAIR-AN, as the degree of hirsutism, fat deposition and menstrual cyclicity were significantly improved. These phenomena could be attributed to the decrease of insulin and androgen circulating levels 
Table 1 Pertinent findings of the patients before and post liraglutide administration.

\begin{tabular}{l}
\hline \\
\hline Weight $(\mathrm{kg})$ \\
BMI $\left(\mathrm{kg} / \mathrm{m}^{2}\right)$ \\
Total Fat (\%) \\
Trunk Fat (\%) \\
Menstrual cycles/year \\
FG-score \\
Cholesterol (mg/dL) \\
HDL (mg/dL) \\
LDL (mg/dL) \\
Triglycerides (mg/dL) \\
Glucose (mg/dL) \\
Insulin (pmol/L) \\
HOMA-IR \\
AUC GLU \\
AUC INS \\
MATSUDA index \\
FSH (IU/L) \\
LH (IU/L) \\
E2 (pg/mL) \\
Testosterone (ng/dL) \\
SHBG (nmol/L) \\
Androstenedione (ng/dL) \\
DHEAS( $\mu$ g/dL) \\
$170 H P(n g / d L)$
\end{tabular}

\begin{tabular}{c}
\hline Before \\
$92.80 \pm 13.78$ \\
$31 \pm 3.51$ \\
$43.12 \pm 4.69$ \\
$42.32 \pm 4.96$ \\
$6 \pm 0.81$ \\
$12.25 \pm 1.25$ \\
$186.51 \pm 10.47$ \\
$41.75 \pm 4.62$ \\
$108.81 \pm 5.12$ \\
$168.3 \pm 13.82$ \\
$85.25 \pm 5.25$ \\
$39.25 \pm 4.820$ \\
$8.388 \pm 1.327$ \\
$15065 \pm 1833$ \\
$23764 \pm 2467$ \\
$1.015 \pm 0.061$ \\
$4.70 \pm 0.63$ \\
$6.52 \pm 2.52$ \\
$49.63 \pm 6.27$ \\
$1.02 \pm 0.08$ \\
$28.50 \pm 0.66$ \\
$3.17 \pm 0.34$ \\
$274 \pm 4.62$ \\
$0.95 \pm 0.052$
\end{tabular}

\begin{tabular}{c}
\hline Post-liraglutide Rx \\
\hline $89.25 \pm 6.10$ \\
$30 \pm 2.83$ \\
$39.25 \pm 1.37$ \\
$37.00 \pm 1.58$ \\
$10.33 \pm 0.88$ \\
$8.75 \pm 0.47$ \\
$172.23 \pm 13.56$ \\
$38.23 \pm 5.72$ \\
$112.64 \pm 6.23$ \\
$161.47 \pm 12.87$ \\
$87.75 \pm 7.00$ \\
$22.44 \pm 5.368$ \\
$5.118 \pm 1.437$ \\
$15818 \pm 3659$ \\
$17723 \pm 3341$ \\
$1.44 \pm 0.25$ \\
$4.55 \pm 0.33$ \\
$5.82 \pm 0.68$ \\
$45.14 \pm 7.64$ \\
$0.63 \pm 0.12$ \\
$25.23 \pm 8.82$ \\
$2.65 \pm 0.49$ \\
$238 \pm 38.43$ \\
$0.84 \pm 0.043$
\end{tabular}

\begin{tabular}{ll}
\hline \multicolumn{1}{c}{$\boldsymbol{P}$} \\
\hline 0.13 \\
0.28 \\
0.035 \\
0.019 \\
0.002 \\
0.003 \\
0.26 \\
0.34 \\
0.28 \\
0.31 \\
0.43 \\
0.0006 \\
0.0003 \\
0.82 \\
0.021 \\
0.25 \\
0.34 \\
0.46 \\
0.62 \\
0.027 \\
0.34 \\
0.03 \\
0.31 \\
0.28 \\
\hline
\end{tabular}

observed in this group of patients. It has to be emphasized that there was no significant weight loss during therapy and, accordingly, the previously mentioned changes should be attributed to liraglutide action per se. In summary, this study provides evidence that liraglutide may be safely and effectively used in the treatment of women suffering from HAIR-AN.

\section{Patient's perspective}

All the patients were very satisfied with their menstrual cycle normalization, the decrease of hyperandrogenic signs and acanthosis nigricans as well as the lack of feeling bloated.

\section{Declaration of interest}

There is no conflict of interest from any author, except from Dr Fotis Papagiannopoulos who works in Novo Nordisk, Greece.

\section{Funding}

This research did not receive any specific grant from any funding agency in the public, commercial or not-for-profit sector.

\section{Patient consent}

Written informed consent was obtained from the patient/patient's mother for publication of this case report.

\section{Author contribution statement}

Livadas $\mathrm{S}$ designed the study, cared for the patients, performed data analysis and wrote the manuscript. Androulakis I, Angelopoulos N, Lytras A and Kassi G cared for the patients. Papagiannopoulos F wrote and edited the manuscript.

\section{References}

1 Rager KM \& Omar HA. Androgen excess disorders in women: the severe insulin-resistant hyperandrogenic syndrome, HAIR-AN. ScientificWorldJournal 20066 116-121. (https://doi.org/10.1100/ tsw.2006.23)

2 Dédjan AH, Chadli A, El Aziz S \& Farouqi A. Hyperandrogenisminsulin resistance-acanthosis nigricans syndrome. Case Reports in Endocrinology 20152015 193097. (https://doi. org/10.1155/2015/193097)

3 Livadas S \& Diamanti-Kandarakis E. Polycystic ovary syndrome: definitions, phenotypes and diagnostic approach. Frontiers of Hormone Research 201340 1-21. (https://doi. org/10.1159/000341673)

4 Macut D, Bjekić-Macut J, Rahelić D \& Doknić M. Insulin and the polycystic ovary syndrome. Diabetes Research and Clinical Practice 2017130 163-170. (https://doi.org/10.1016/j.diabres.2017.06.011)

5 Buse JB, Rosenstock J, Sesti G, Schmidt WE, Montanya E, Brett JH, Zychma M \& Blonde L. LEAD-6 Study Group. Liraglutide once a day versus exenatide twice a day for type 2 diabetes: a 26-week randomized, parallel-group, multinational, open-label trial (LEAD-6). Lancet 2009374 39-47. (https://doi.org/10.1016/S01406736(09)60659-0)

6 Jensterle M, Kocjan T, Pfeifer M, Kravos NA \& Janez A. Shortterm combined treatment with liraglutide and metformin leads to significant weight loss in obese women with polycystic ovary 
syndrome and previous poor response to metformin. European Journal of Endocrinology 2014170 451-459. (https://doi.org/10.1530/EJE-130797)

7 Elkind-Hirsch K, Marrioneaux O, Bhushan M, Vernor D \& Bhushan R. Comparison of single and combined treatment with exenatide and metformin on menstrual cyclicity in overweight women with polycystic ovary syndrome. Journal of Clinical Endocrinology and Metabolism 200893 2670-2678. (https://doi. org/10.1210/jc.2008-0115)

8 Tzotzas T, Karras SN \& Katsiki N. Glucagon-like peptide-1 (GLP-1) receptor agonists in the treatment of obese women with polycystic ovary syndrome. Current Vascular Pharmacology 201715 218-229. (https://doi.org/10.2174/1570161114666161221115324)

9 Lamos EM, Malek R \& Davis SN. GLP-1 receptor agonists in the treatment of polycystic ovary syndrome. Expert Review of Clinical Pharmacology 201710 401-408. (https://doi.org/10.1080/17512433.2 017.1292125)

10 Li CJ, Yu Q, Yu P, Yu TL, Zhang QM, Lu S \& Yu DM. Changes in liraglutide induced body composition are related to modifications in plasma cardiac natriuretic peptides levels in obese type 2 diabetic patients. Cardiovascular Diabetology 201413 36. (https://doi. org/10.1186/1475-2840-13-36)

Received in final form 5 March 2020

Accepted 15 May 2020 